\title{
Sampling frequency impacts measurement of walking activity after stroke
}

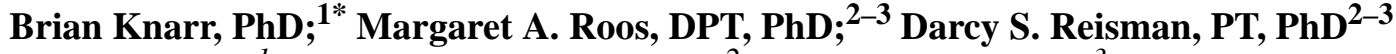 \\ Departments of ${ }^{1}$ Mechanical Engineering and ${ }^{2}$ Physical Therapy, and ${ }^{3}$ Interdisciplinary Graduate Program in Biome- \\ chanics and Movement Science, University of Delaware, Newark, DE
}

\begin{abstract}
The purpose of this study was to examine the effect of sampling epoch on total time spent walking and number of walking bouts per day in persons with stroke. Ninety-eight persons with average age of $63.8 \mathrm{yr}$ and average time poststroke of 43.6 mo participated. Participants wore a StepWatch Activity Monitor for 3 to 5 consecutive days. The number of strides taken was collected in consecutive $5 \mathrm{~s}$ epochs and down sampled into 10, 20, 30, and $60 \mathrm{~s}$ epochs. Total time walking and total number of walking bouts were determined for each day. Low activity days were defined as days below the 25th percentile of total steps per day and high activity days as days above the 75th percentile of total steps per day. Total time walking and total number of bouts were different for each sampling epoch ( $p<0.001$ for all). The 5 s sampling epoch resulted in calculation of $\sim 40 \%$ of the walking time and $\sim 6$ times as many bouts as a $60 \mathrm{~s}$ sampling epoch. Differences were greater for low activity days ( $p<0.001$ for all). Sampling epoch affects daily step activity variables whose calculation depends on time, especially during low activity days. Sampling epoch must be carefully considered when designing studies aimed at understanding patterns of daily walking activity.
\end{abstract}

Key words: ambulation, CVA, hemiparesis, hemiplegia, locomotor activity, neurological disorders, physical activity, physical exercise, stroke, walking.

\section{INTRODUCTION}

Approximately 80 percent of the 5.5 million people living with stroke have some level of disability [1]. This disability is a consequence of, and a risk factor for, physi- cal inactivity [2-3]. Lack of physical activity has serious health and functional consequences for people after stroke [4]. Therefore, a thorough and accurate measurement of inactivity after stroke is necessary to develop optimal interventions to improve activity.

To obtain objective measurement of walking activity after stroke, many studies utilize an accelerometry-based system [5]. Several studies have established the reliability and accuracy of these devices in persons with stroke [6-8] and have emphasized the importance of the ability to adjust measurement properties of the device (e.g., sensitivity to motion) to enhance accurate measurement [67]. One measurement property, however, that has not received systematic investigation is the sampling epoch. While the length of the sampling epoch will not affect the calculation of steps per day, as studies begin to analyze step activity data in a more detailed way [9-10], the length of the sampling epoch becomes critical [11-12]. For example, the calculation of the amount of time spent walking is highly dependent upon sampling epoch. Consider two studies of walking activity after stroke. In one study, the sampling interval is set to $60 \mathrm{~s}$ [13], and in the other, it is set to $15 \mathrm{~s}$ [14]. Now imagine that a participant

\footnotetext{
Abbreviations: ANOVA $=$ analysis of variance, SAM = StepWatch Activity Monitor.

*Address all correspondence to Brian A. Knarr, PhD; University of Delaware, 126 Spencer Lab, Newark, DE 19716; 908-380-4413. Email: bknarr@udel.edu
}

http://dx.doi.org/10.1682/JRRD.2012.12.0225 
in each study took several steps in a row, amounting to $7 \mathrm{~s}$ of continuous walking, and then sat down. In the study where the sampling epoch was set to $60 \mathrm{~s}$, the subject would be given credit for $60 \mathrm{~s}$ of walking, because the system cannot detect time intervals shorter than $60 \mathrm{~s}$. In contrast, in the study where the sampling epoch was set to $15 \mathrm{~s}$, the subject would be given credit for $15 \mathrm{~s}$ of walking, much closer to the actual time spent walking. This overestimation of time spent walking when using a longer sampling epoch is particularly problematic in calculations aimed at comparing different groups of subjects. The purpose of this study was to systematically examine the effect of sampling epoch on total time spent walking and number of walking bouts per day in persons with stroke. We hypothesized that the use of longer sampling epochs would overestimate the time spent walking and underestimate the number of discrete walking bouts and that the magnitude of this over- and underestimation due to longer sampling epochs would be greater when the number of steps per day was low.

\section{METHODS}

\section{Participants}

Individuals who were over the age of 18 , were living in the community, and had experienced one or more strokes participated. Individuals were excluded if they had additional neurologic diagnoses, were unable to walk without assistance from another person (orthotics and assistive devices allowed), or were unable to follow instruction or communicate with investigators.

\section{Procedures}

A StepWatch Activity Monitor (SAM) (Orthocare Innovations; Seattle, Washington) was calibrated to the subject's height and weight and placed above the subject's ankle on the nonparetic leg. Calibration was checked with 30 strides at a subject's self-selected walking speed and with 10 strides at a faster speed. The SAM was recalibrated if the stride count differed by $>2$ between the SAM and manual counting. Participants wore the SAM during all waking hours (except bathing and swimming) for 3-5 consecutive days. The number of strides taken was collected in consecutive 5 s epochs.

\section{Data Analysis}

Before data analysis, all data from the SAM was reviewed to eliminate partial days (e.g., days when the subject did not wear the SAM during all waking hours). The SAM data were processed using a custom-written MATLAB program (MathWorks; Natick, Massachusetts). Data recorded by the SAM in strides were converted to steps for analysis within the program by multiplying by a factor of 2. Data collected in $5 \mathrm{~s}$ epochs were down sampled into 10, 20, 30, and 60 s sampling epochs to investigate the effect of sampling frequency. For each day collected, the total time walking and the total number of walking bouts were calculated at each of the five sampling rates. Total time walking was calculated as the total number of sampling epochs during which at least one stride was recorded multiplied by the length of the sampling epoch. The total number of walking bouts was calculated as the sum of discrete walking bouts, with the start of a walking bout defined as a sampling epoch with activity (nonzero) and the end of a bout defined as a sampling epoch with no activity (zero strides).

Data were normalized to the 60 s sampling epoch. A repeated measures analysis of variance (ANOVA) was used to assess differences between the five sampling rates for both the normalized time walking and the normalized number of bouts. Post hoc paired $t$-tests were performed to assess significance between individual sampling epochs.

For analysis of the influence of amount of walking activity on the relationship between sampling rates, days in which the total number of steps fell below the 25th percentile were classified as low activity days. Similarly, days in which the total number of steps was greater than the 75th percentile were classified as high activity days. Post hoc paired $t$-tests were run comparing the time walking and number of bouts between low and high activity days for all sampling epochs. The significance level for the repeated measures ANOVA was set at 0.05. A Bonferroni correction was applied for the post hoc paired $t$-test with $10 \mathrm{com}$ parisons, yielding a significance level of 0.005 .

\section{RESULTS}

Ninety-eight subjects (59 male) with average (mean \pm standard deviation) age of $63.8 \pm 10.3 \mathrm{yr}$, gait speed of $0.67 \pm 0.29 \mathrm{~m} / \mathrm{s}$, and time poststroke of $43.6 \pm 58.1 \mathrm{mo}$ participated in this study, resulting in a total of $366 \mathrm{~d}$ sampled. 
Subjects walked an average of 5,432 $\pm 3,727$ steps/d. Total time walking and total number of bouts were different for each sampling epoch ( $p<0.001$ for all cases). The $5 \mathrm{~s}$ sampling epoch resulted in calculation of $\sim 40$ percent of the walking time of a $60 \mathrm{~s}$ sampling epoch (Figure 1(a), Table 1) and $\sim 6$ times as many bouts as a $60 \mathrm{~s}$ sampling epoch (Figure 2(a)).

When low activity ( $<2,500$ steps, $n=93)$ and high activity ( $>7,500$ steps, $n=86$ ) days were compared, the percent change in walking time and number of bouts was greater for the low activity days from the $5 \mathrm{~s}$ sampling epoch to each subsequent higher sampling epoch (all comparisons $p<0.001$, Figures 1(b) and 2(b)). This indicates that the over- and underestimation that occurred with the longer sampling epochs were worse for the low activity days.

\section{DISCUSSION}

The results of this study show that the effect of the length of the sampling epoch differed based on the amount of walking activity performed. The effect of sampling epoch was greater for the days with low activity $(<2,500$ steps) than for those with high activity $(>7,500$ steps). The result of this is that differences between groups with largely different number of steps per day would be compressed when larger sampling epochs are chosen (Figures 1(c) and 2(c)). This has potential implications for the interpretation of step activity data comparisons between groups of subjects with largely different number of steps per day, such as sedentary and active older adults [15] or adults with stroke and neurologically intact adults [9].

The selection of epoch size is also important when comparing subjects who take a similar number of steps per day, but have different activity patterns. As an example, the analysis of two subjects who walked a similar number of steps in a day is shown in Table 2 . When $60 \mathrm{~s}$ epochs are used to analyze their walking patterns, it is calculated that subject $\mathrm{Y}$ walked for $1 \mathrm{~h}$ longer than subject $X$ in only 9 more bouts (20\% more bouts). In contrast, when the data are analyzed with $5 \mathrm{~s}$ bouts, it is calculated that subject $\mathrm{Y}$ walked only $\sim 8 \mathrm{~min}(0.13 \mathrm{~h})$ longer than subject $X$, while participating in 110 more bouts (35\% more bouts). For these subjects, the selection of epoch duration has a meaningful influence on the subjects' (a)

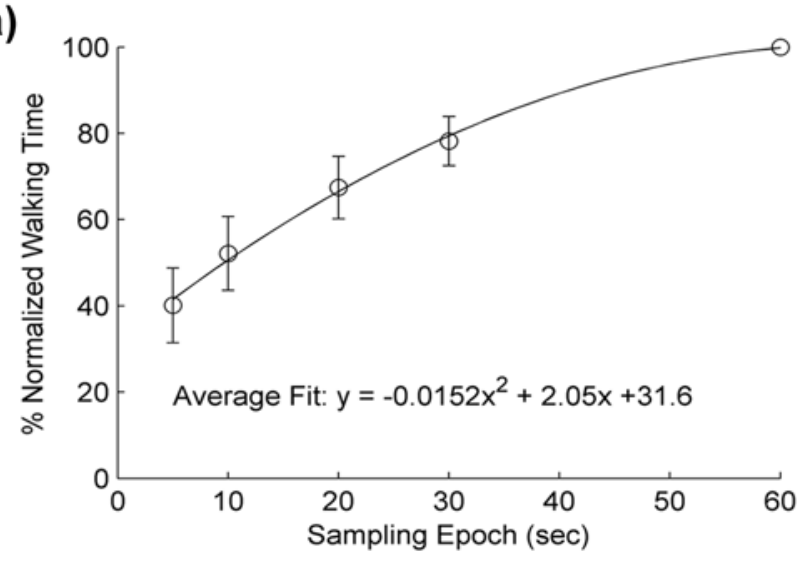

(b)

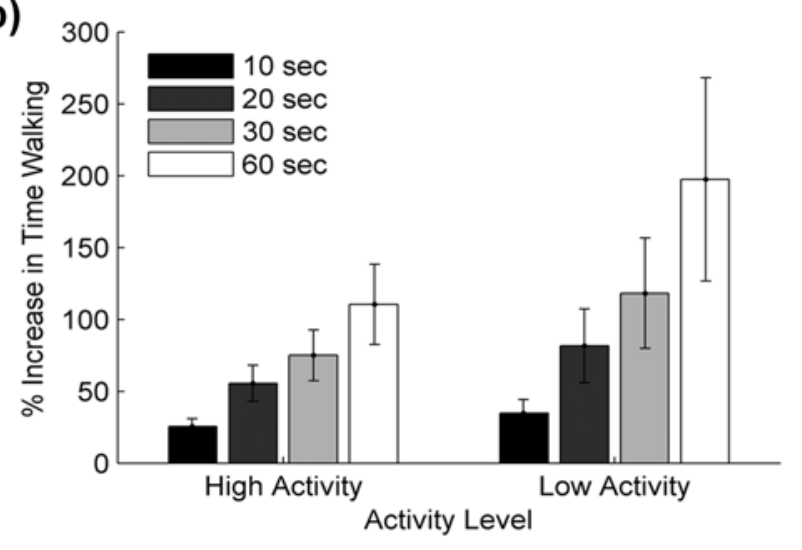

(c)

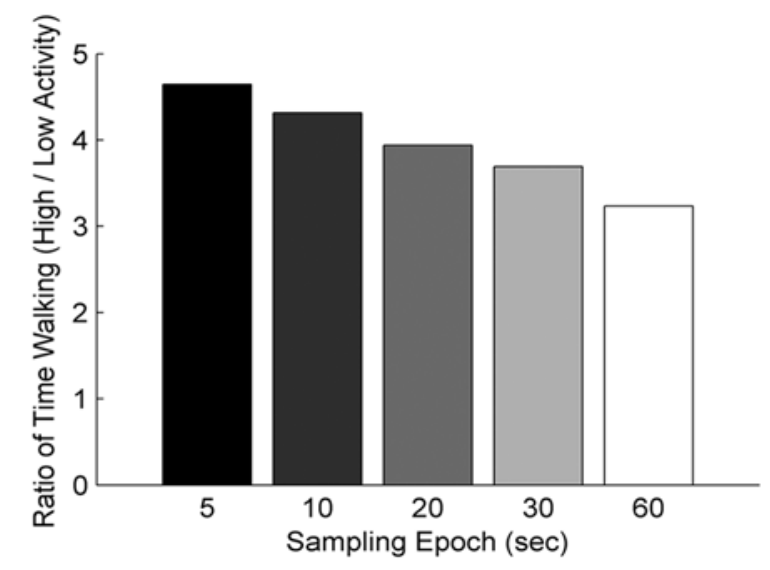

Figure 1.

(a) Time spent walking per day normalized to time calculated using $60 \mathrm{~s}$ sampling epochs. Significant differences were found between all sampling epochs. (b) Percent increase in time walking per day compared with $5 \mathrm{~s}$ sampling epoch in high activity ( $>7,500$ steps/d) and low activity ( $<2,500$ steps/d) days. (c) Ratio of time walking between high and low activity days at all sampling epochs. Notice that difference between high and low activity days is reduced with each subsequently larger sampling epoch. 
Table 1.

Calculated time spent walking and total number of bouts per day for five epoch lengths analyzed. Data shown as mean \pm standard deviation.

\begin{tabular}{ccc}
\hline $\begin{array}{c}\text { Bout } \\
\text { Length (s) }\end{array}$ & $\begin{array}{c}\text { Time } \\
\text { Walking/d (h) }\end{array}$ & No. of Bouts/d \\
\hline 5 & $1.65 \pm 0.97$ & $356.25 \pm 196.40$ \\
10 & $2.14 \pm 1.21$ & $223.77 \pm 119.55$ \\
20 & $2.76 \pm 1.50$ & $135.31 \pm 67.63$ \\
30 & $3.18 \pm 1.68$ & $100.08 \pm 46.92$ \\
60 & $4.02 \pm 2.00$ & $60.22 \pm 25.51$ \\
\hline \hline
\end{tabular}

calculated activity level, despite walking a similar number of steps.

Many studies that examine daily step activity using an accelerometry-based device in persons with stroke do not report the sampling epoch used [7,16-17] and may use the default epoch set by the company (e.g., $60 \mathrm{~s}$ epoch for the SAM device). While this choice may be appropriate for measurement of steps per day, the results of this study suggest that it may not be optimal when examining the time spent walking or number of bouts per day. Moreover, other measurements, such as step intensity [10], where the time interval is important in the calculation, are also likely affected by the choice of sampling epoch. A measurement such as cadence, which describes the rate of steps taken, is highly dependent on sampling epoch. For example, if an individual takes 5 steps in a $5 \mathrm{~s}$ epoch and rests for $1 \mathrm{~min}$, he or she would have a cadence of $1 \mathrm{step} / \mathrm{s}$ during the $5 \mathrm{~s}$ epoch of activity. If the data were collected in $60 \mathrm{~s}$ epochs, however, those same 5 steps would result in a cadence of 5 steps/60 $\mathrm{s}$, or $0.083 \mathrm{steps} / \mathrm{s}$, a much lower cadence for the same activity. For some devices, longer sampling epochs result in the storage of a fewer number of days of walking [11$12]$, so it is necessary to strike a balance between recording an adequate number of days of walking [18] and the need to use an appropriate sampling frequency.

\section{CONCLUSIONS}

The length of the sampling epoch affects the calculation of daily step activity variables whose calculation depends implicitly or explicitly on time. The size of this effect varies depending on the amount of daily step activity. Therefore, the sampling epoch must be carefully considered when designing studies aimed at understanding patterns of daily walking activity.
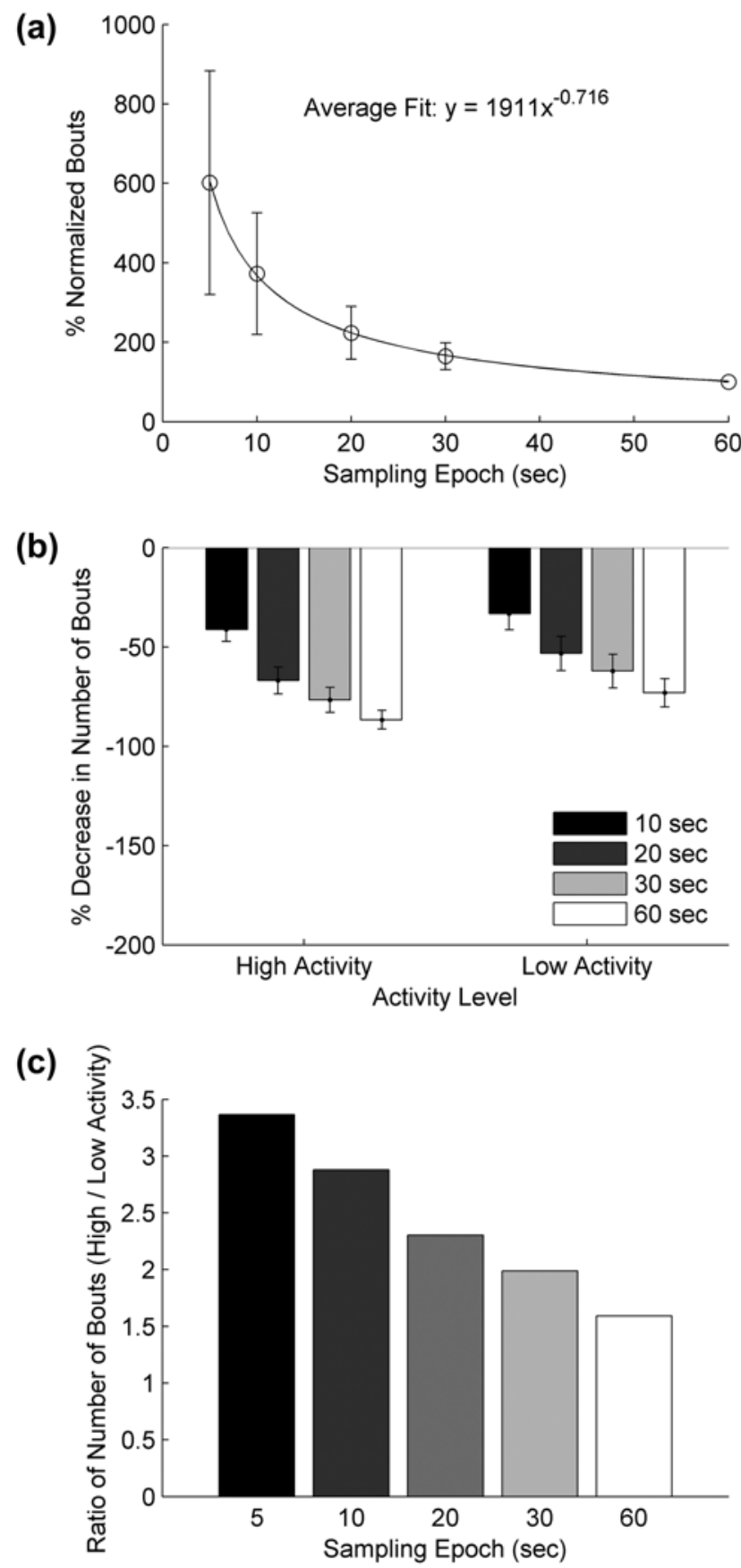

Figure 2.

(a) Number of walking bouts per day normalized to $60 \mathrm{~s}$ sampling epoch. Significant differences were found between all sampling epochs. (b) Percent decrease in number of bouts per day compared with $5 \mathrm{~s}$ sampling epoch in high activity $(>7,500$ steps/d) and low activity ( $<2,500$ steps/d) days. (c) Ratio of number of bouts between high and low activity days at all sampling epochs. Notice that difference between high and low activity days is reduced with each subsequently larger sampling epoch. 
Table 2.

Example day of activity for two subjects, $\mathrm{X}$ and $\mathrm{Y}$, with similar steps within day. Steps, time walking, and number of bouts for day across five epoch lengths analyzed.

\begin{tabular}{|c|c|c|c|c|c|c|}
\hline \multirow{2}{*}{ Bout Length (s) } & \multicolumn{2}{|c|}{ Steps } & \multicolumn{2}{|c|}{ Time Walking (h) } & \multicolumn{2}{|c|}{ No. of Bouts } \\
\hline & $\mathbf{X}$ & $\mathbf{Y}$ & $\mathbf{X}$ & $\mathbf{Y}$ & $\mathbf{X}$ & $\mathbf{Y}$ \\
\hline 5 & 4,778 & 4,858 & 1.45 & 1.58 & 308 & 418 \\
\hline 10 & 4,778 & 4,858 & 1.87 & 2.16 & 179 & 266 \\
\hline 20 & 4,778 & 4,858 & 2.34 & 2.92 & 106 & 150 \\
\hline 30 & 4,778 & 4,858 & 2.72 & 3.43 & 74 & 114 \\
\hline 60 & 4,778 & 4,858 & 3.35 & 4.35 & 53 & 64 \\
\hline
\end{tabular}

\section{ACKNOWLEDGMENTS}

\author{
Author Contributions: \\ Conceived study: D. S. Reisman. \\ Collected data: M. A. Roos. \\ Processed data: B. Knarr. \\ Analyzed data: B. Knarr, M. A. Roos, D. S. Reisman. \\ Prepared manuscript: B. Knarr, D. S. Reisman. \\ Edited manuscript: M. A. Roos.
}

Financial Disclosures: The authors have declared that no competing interests exist.

Funding/Support: This material was based on work supported by an Institutional Development Award from the National Institute of General Medical Sciences of the National Institutes of Health (grant P30GM103333-01) and the National Institutes of Health (grants NR010786 and R21HD07142).

Institutional Review: All included individuals signed informed consent approved by the Human Subjects Review Board at University of Delaware before participation.

Participant Follow-Up: The authors do not plan to inform participants of the publication of this study.

\section{REFERENCES}

1. Lees KR, Zivin JA, Ashwood T, Davalos A, Davis SM, Diener HC, Grotta J, Lyden P, Shuaib A, Hårdemark HG, Wasiewski WW; Stroke-Acute Ischemic NXY Treatment (SAINT I) Trial Investigators. NXY-059 for acute ischemic stroke. N Engl J Med. 2006;354(6):588-600.

[PMID:16467546]

http://dx.doi.org/10.1056/NEJMoa052980

2. Mayo NE, Wood-Dauphinee S, Côté R, Durcan L, Carlton J. Activity, participation, and quality of life 6 months poststroke. Arch Phys Med Rehabil. 2002;83(8):1035-42.

[PMID:12161823]

http://dx.doi.org/10.1053/apmr.2002.33984

3. Rimmer JH, Wang E. Aerobic exercise training in stroke survivors. Top Stroke Rehabil. 2005;12(1):17-30.
[PMID:15735998]

http://dx.doi.org/10.1310/L6HG-8X8N-QC9Q-HHM8

4. Hornnes N, Larsen K, Boysen G. Little change of modifiable risk factors 1 year after stroke: a pilot study. Int J Stroke. 2010;5(3):157-62.

5. Gebruers N, Vanroy C, Truijen S, Engelborghs S, De Deyn PP. Monitoring of physical activity after stroke: a systematic review of accelerometry-based measures. Arch Phys Med Rehabil. 2010;91(2):288-97. [PMID:20159136] http://dx.doi.org/10.1016/j.apmr.2009.10.025

6. Macko RF, Haeuber E, Shaughnessy M, Coleman KL, Boone DA, Smith GV, Silver KH. Microprocessor-based ambulatory activity monitoring in stroke patients. Med Sci Sports Exerc. 2002;34(3):394-99. [PMID:11880800] http://dx.doi.org/10.1097/00005768-200203000-00002

7. Mudge S, Stott NS. Test-retest reliability of the StepWatch Activity Monitor outputs in individuals with chronic stroke. Clin Rehabil. 2008;22(10-11):871-77. [PMID:18955419] http://dx.doi.org/10.1177/0269215508092822

8. Mudge S, Stott NS, Walt SE. Criterion validity of the StepWatch Activity Monitor as a measure of walking activity in patients after stroke. Arch Phys Med Rehabil. 2007;88(12):1710-15. [PMID:18047890] http://dx.doi.org/10.1016/j.apmr.2007.07.039

9. Roos MA, Rudolph KS, Reisman DS. The structure of walking activity in people after stroke compared with older adults without disability: a cross-sectional study. Phys Ther. 2012;92(9):1141-47. [PMID:22677293] http://dx.doi.org/10.2522/ptj.20120034

10. Manns PJ, Baldwin E. Ambulatory activity of stroke survivors: measurement options for dose, intensity, and variability of activity. Stroke. 2009;40(3):864-67.

[PMID:19150867]

http://dx.doi.org/10.1161/STROKEAHA.108.531590

11. Coleman KL, Smith DG, Boone DA, Joseph AW, del Aguila MA. Step activity monitor: long-term, continuous recording of ambulatory function. J Rehabil Res Dev. 1999; 36(1):8-18. [PMID:10659890] 
12. Matthews CE, Hagströmer M, Pober DM, Bowles HR. Best practices for using physical activity monitors in population-based research. Med Sci Sports Exerc. 2012;44(1, Suppl 1):S68-76. [PMID:22157777]

13. Hale LA, Pal J, Becker I. Measuring free-living physical activity in adults with and without neurologic dysfunction with a triaxial accelerometer. Arch Phys Med Rehabil. 2008;89(9):1765-71. [PMID:18760161] http://dx.doi.org/10.1016/j.apmr.2008.02.027

14. Rand D, Eng JJ, Tang PF, Jeng JS, Hung C. How active are people with stroke? Use of accelerometers to assess physical activity. Stroke. 2009;40(1):163-68. [PMID:18948606] http://dx.doi.org/10.1161/STROKEAHA.108.523621

15. Cavanaugh JT, Coleman KL, Gaines JM, Laing L, Morey MC. Using step activity monitoring to characterize ambulatory activity in community-dwelling older adults. J Am Geriatr Soc. 2007;55(1):120-24. [PMID:17233695] http://dx.doi.org/10.1111/j.1532-5415.2006.00997.x

16. Bowden MG, Balasubramanian CK, Behrman AL, Kautz SA. Validation of a speed-based classification system using quantitative measures of walking performance poststroke. Neurorehabil Neural Repair. 2008;22(6):672-75. [PMID:18971382] http://dx.doi.org/10.1177/1545968308318837
17. Mudge S, Stott NS. Timed walking tests correlate with daily step activity in persons with stroke. Arch Phys Med Rehabil. 2009;90(2):296-301. [PMID:19236983] http://dx.doi.org/10.1016/j.apmr.2008.07.025

18. Tudor-Locke C, Burkett L, Reis JP, Ainsworth BE, Macera CA, Wilson DK. How many days of pedometer monitoring predict weekly physical activity in adults? Prev Med. 2005;40(3):293-98. [PMID:15533542] http://dx.doi.org/10.1016/j.ypmed.2004.06.003

Submitted for publication December 11, 2012. Accepted in revised form February 4, 2013.

This article and any supplemental materials should be cited as follows:

Knarr B, Roos MA, Reisman DS. Sampling frequency impacts measurement of walking activity after stroke. J Rehabil Res Dev. 2013;50(8):1107-12.

http://dx.doi.org/10.1682/JRRD.2012.12.0225

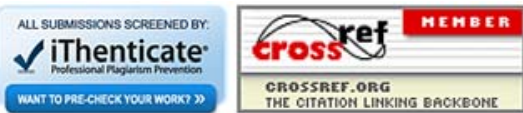

